\title{
Evaluación de la Dureza Superficial en Amalgamas en Función de Tipos de Aleaciones, Momentos y Tipos de Pulimentos.
}

\author{
Juliana Panazzolo Ramos ${ }^{\mathrm{a}}$, Alma Blásida Concepción Elizaur Benitez Catirse ${ }^{\mathrm{b}}$, \\ Silmara Aparecida Milori Corona ${ }^{c}$ \\ aÁrea de Rehabilitación Oral, Universidade de São Paulo, \\ Faculdade de Odontologia de Ribeirão Preto \\ Avenida do Café, s/n. 14040-904 Ribeirão Preto - SP, Brasil \\ ${ }^{\mathrm{b}}$ Departamento de Materiales Dentários y Prótesis, Universidade de São Paulo, \\ Faculdade de Odontologia de Ribeirão Preto \\ ${ }^{\mathrm{c}}$ Departamento Dentistica Restauradora, Universidade de São Paulo, \\ Faculdade de Odontologia de Ribeirão Preto
}

Received: December 30, 2002; Revised: May 1, 2003

\begin{abstract}
The aim of this study was to analyze in vitro surface hardness (micro-indentation tester Wolpert) of different types of amalgam alloys varying the technique and polishing time. Tested were the amalgam: Velvalloy, Permite e Dispersalloy. For each material, 2 polishing techniques were accomplished: $\mathrm{T}_{1}$ (bladed finishing burs + abrasive rubber points + pumice-water slurry in Robinson polishing brush) and; $\mathrm{T}_{2}$ (abrasive rubber points + pumice-water slurry in Robinson polishing brush), at 2 periods of time: $\mathrm{P}_{1}$ (immediately - 15 min after condensation) and $\mathrm{P}_{2}$ (after $24 \mathrm{~h}$ ). For each experimental condition, 5 samples were prepared. For each sample, 2 indentations were taken and the average was determined. Were made analysis using ANOVA and Tukey test. Were concluded that: a) using the Permite alloy with polishing after $24 \mathrm{~h}$ the surface hardness of restoration will be higher; $b$ ) the technique $\mathrm{T}_{2}$ enhances the hardness of dental amalgams and c) alloy Permite with polished immediately were statisticaly equal than Velvalloy polished after $24 \mathrm{~h}$.
\end{abstract}

Keywords: amalgam, surface hardness, polishing, repolishing

\section{Introducción}

La dureza superficial de la amalgama está directamente relacionada con la velocidad de cristalización de este material y por consecuencia, influye de modo directo en la elección del momento y técnicas de pulimento.

El pulimento de las restauraciones es un procedimiento de suma importancia, afectando vários aspectos de una restauración de amalgama, tales como longevidad, estética y biocompatibilidad con los tejidos bucales (Fenton \& Smales, 1984; Drummond et al., 1992), así como también disminuye la liberación de mercurio en la cavidad bucal (Elizaur et al., 1987). Diversos estudios afirman que una superfície pulida aumenta la resistencia a la corrosión, compresión y a las fracturas marginales (Garone Neto, 1981; Sundfeld et al., 1989; Yamashita et al., 1995; Canilo et al., 1996; Anusavice, 1998).
Al margen de eso, el própio pulimento influye en la dureza superficial de la amalgama conjuntamente con la seleción del tipo de aleación.

A pesar de que el pulimento ofrece a las restauraciones de amalgamas benefícios fundamentales, muchos odontólogos no lo realizan, justificando esta negligencia por la pérdida de tiempo que su ejecución representa, calor generado durante el procedimiento, falta de practicidad, gran cantidad de técnicas (Nitkin \& Goldberg, 1979; Yamashita et al., 1995; Pereira et al., 1998), que frecuentemente, generan dudas con relación a la elección y momento adecuado de su ejecución. Pues hay siempre una mayor preocupación con el aspecto metalográfico de la restauración.

Siendo así, en este trabajo fue realizado la evaluación de la dureza superficial, en función de dos técnicas de pulimento realizadas en dos momentos distintos, utilizando para eso 3 aleaciones para amalgama.

*e-mail: alma@forp.usp.br 


\section{Materiales y Método}

Para la confección de los especímenes, la amalgama fue condensada en cavidades de $5 \mathrm{~mm}$ de diámetro por $2 \mathrm{~mm}$ de altura contenidas en una base de resina acrílica.

La relación aleación/mercúrio para la aleación Permite fue de 1:0,92, obtenida por el uso de cápsulas pre-adosadas provistas por el fabricante, trituradas en amalgamador mecánico Ultramatic - SDI. Para la aleación Dispersalloy y Velvalloy fue establecida la proporción de 1:1 y triturado en amalgamador Dosamix - Dabi Atlante (Tabla 1).

Para cada tipo de aleación para amalgama fueron confeccionados 20 especímenes, siendo que 10 fueron sometidas a pulimento inmediato $\left(\mathrm{P}_{1}\right)$ y 10 a pulimento después de $24 \mathrm{~h}\left(\mathrm{P}_{2}\right)$ y de estos 10, cinco recibían pulimento utilizando la técnica $\mathrm{T}_{1}$ y cinco la técnica $\mathrm{T}_{2}$ Así fueron realizadas cinco repeticiones para cada condición experimental y un total de 60 especímenes.

La amalgama fue condensada utilizando condensadores de Ward, con diámetros en orden creciente, en pequeñas porciones hasta llenar en exceso la cavidad. Este exceso fue removido con un instrumento cortante, con la finalidad de obtener una superficie plana con relación a los bordes de la matriz (Elizaur et al., 1987).

Seguidamente los especímenes fueron sometidos a pulimentos de acuerdo con las técnicas establecidas, siendo ellas: $\mathrm{T}_{1}$ : Brocas multilaminadas en forma de pera (Bush) + Gomas abrasivas en forma de cono (Viking - KG Sorensen) en orden decreciente de abrasividad + piedra pómez con agua aplicada con escoba de Robinson (Viking - KG Sorensen); $\mathrm{T}_{2}$ : Gomas abrasivas en orden decreciente de abrasividad + piedra pómez con agua aplicada con escoba de Robinson. La piedra pomes utilizada presentaba granulación de aproximadamente $37 \mu \mathrm{m}$ (obtenida con el auxilio de Tamiz de $400 \mathrm{Mash}$ ), y portanto granulación menor que de la goma abrasiva azul (de $44 \mu \mathrm{m})$.

El pulimento fue realizado en dos momentos, $\mathrm{P}_{1}: 15 \mathrm{~min}$ después a la condensación y $\mathrm{P}_{2}: 24$ h después de la condensación.

Los especímenes pulidos después de $24 \mathrm{~h}$ fueron inmersos en agua destilada hasta el momento de la realización del pulimento, y almacenados en estufa a la temperatura de $37 \pm 1{ }^{\circ} \mathrm{C}$. Una vez pulidos fueron realizadas lecturas de dureza superficial, utilizándose el microdurómetro Wolpert-Werke (procedencia alemana).
Para cada espécimen fueron realizadas dos lecturas inmediatamente después del tratamiento superficial, resultando en un total de 120 medidas. Los datos fueron sometidos a un análisis de variancia (Tabla 2) que mostró haber significancia $(\mathrm{p} \leq 0,05)$, para los factores estudiados y para la interacción Material $(\mathrm{M}) \times$ Momento de Pulimento $(\mathrm{P})$. También fue aplicado el test complementario de Tukey para verificar las diferencias.

\section{Resultados}

Para el factor Material (M), se verificó que las amalgamas confeccionadas con las aleación Velvalloy $\left(\mathrm{M}_{1}=39,1 \mathrm{VHN}\right)$ presentaron una menor dureza superficial, mientras que los de la aleación Permite $\left(\mathrm{M}_{3}=58,9 \mathrm{VHN}\right)$ determinaron una mayor dureza superficial, permaneciendo la de Dispersalloy $\left(\mathrm{M}_{2}=50,1 \mathrm{VHN}\right)$ en posición intermédia (Fig. 1). El valor crítico de Tukey fue de 7,3 para este factor y del desvío padrón fue de 1,68.

La Técnica $\mathrm{T}_{1}$ que emplea el uso de brocas multilaminadas, gomas abrasivas y piedra pomes (46,8 VHN) determinó una menor dureza que la técnica $\mathrm{T}_{2}$, que utiliza gomas abrasivas y piedra pomes (51,8 VHN) (Fig. 2). Para este factor el desvío padrón fue de 1,37 y el valor crítico de Tukey de 3,92.

Las amalgamas sometidas al pulimento inmediato $\left(\mathrm{P}_{1}=40.5 \mathrm{VHN}\right)$ determinaron menores valores de dureza superficial que cuando comparados con el pulimento realizado después de las 24 h ( $\mathrm{P}_{2}=58.1$ VHN) (Fig. 3). El desvío padrón fue de 1,35 y el valor crítico de Tukey de 3,92.

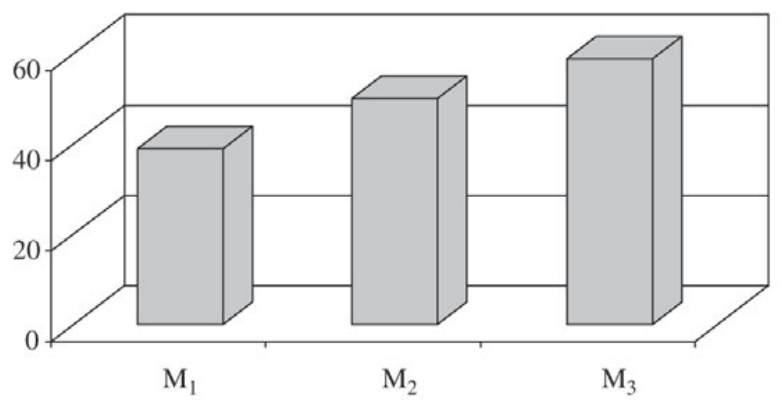

Figura 1. Factor material (M).

Tabla 1. Aleaciones utilizadas.

\begin{tabular}{lcccc}
\hline Sigla & Marca Comercial & Tipo de Aleación & Lote & Fabricante \\
\hline$M_{1}$ & Velvalloy & Convencional & ORE & S.S.White \\
$M_{2}$ & Dispersalloy & Fase Dispersa & 79705161E & Dentsply \\
$M_{3}$ & Permite & Esférica + Convencional & 6092021 & SDI \\
\hline
\end{tabular}


Tabla 2. Resumen del analisis de variancia para dureza superficial (VHN).

\begin{tabular}{lccccc}
\hline Fuente de variación & $\begin{array}{c}\text { Suma de los } \\
\text { Cuadrados }\end{array}$ & $\begin{array}{c}\text { Grados de } \\
\text { Liberdad }\end{array}$ & $\begin{array}{c}\text { Cuadrados } \\
\text { medios }\end{array}$ & $(\mathrm{F})$ & Probabilidad $\left(\mathrm{H}_{0}\right)$ \\
\hline Técnica $(\mathrm{T})$ & 365,33 & 1 & 365,33 & 6,40 & $1,40 \%$ \\
Material $(\mathrm{M})$ & 3947,06 & 2 & 1973,53 & 34,57 & $0,00 \%$ \\
Pulimento $(\mathrm{P})$ & 4680,79 & 1 & 4680,79 & 82,00 & $0,00 \%$ \\
Interacción T $\times \mathrm{M}$ & 66,84 & 2 & 33,42 & 0,59 & $43,42 \%$ \\
Interacción M $\times \mathrm{P}$ & 387,78 & 2 & 193,89 & 3,40 & $4,05 \%$ \\
Interacción T $\times \mathrm{P}$ & 2,26 & 1 & 2,26 & 0,04 & $16,29 \%$ \\
Interacción T $\times \mathrm{M} \times \mathrm{P}$ & 59,94 & 2 & 2,26 & 0,53 & $39,96 \%$ \\
Resíduo & 1739,90 & 48 & 57,08 & & \\
Variación Total & 12250,01 & 59 & & & \\
\hline
\end{tabular}

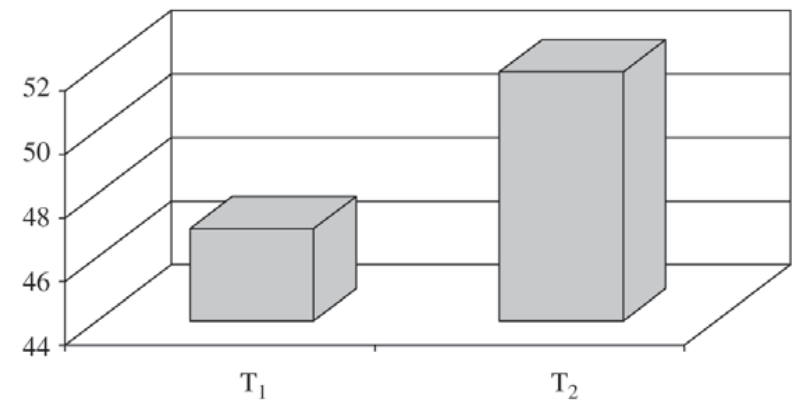

Figura 2. Factor técnica de pulimento (T).

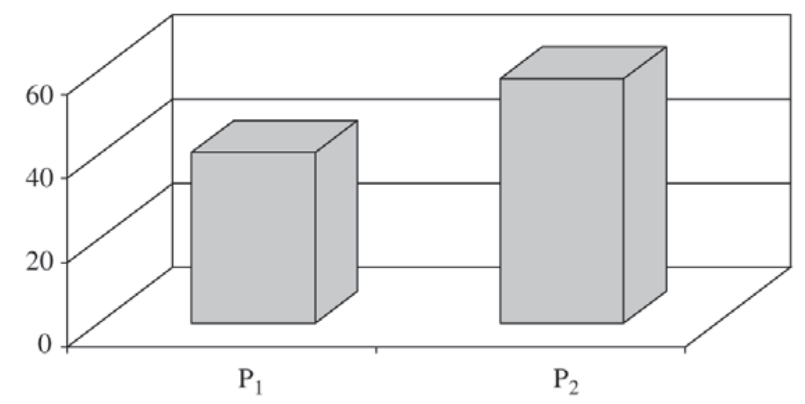

Figura 3. Factor momento de pulimento $(\mathrm{P})$.

Analizando el Fig. 4 de medias de dureza superficial para la interacción Material $(\mathrm{M}) \times$ Momento de Pulimento (P), cuyo dp fue de 2,38 y el valor de Tukey fue de 10,03, se constata que todas las aleaciones estudiadas, cuando sometidas al pulimento inmediato $\left(\mathrm{P}_{1}\right)$ presentaron menor dureza superficial que cuando sometidas al pulimento después de las $24 \mathrm{~h}\left(\mathrm{P}_{2}\right)$. La aleación Permite pulido inmediatamente $(49,7 \mathrm{VHN})$ determinó mayor media de dureza superfi-

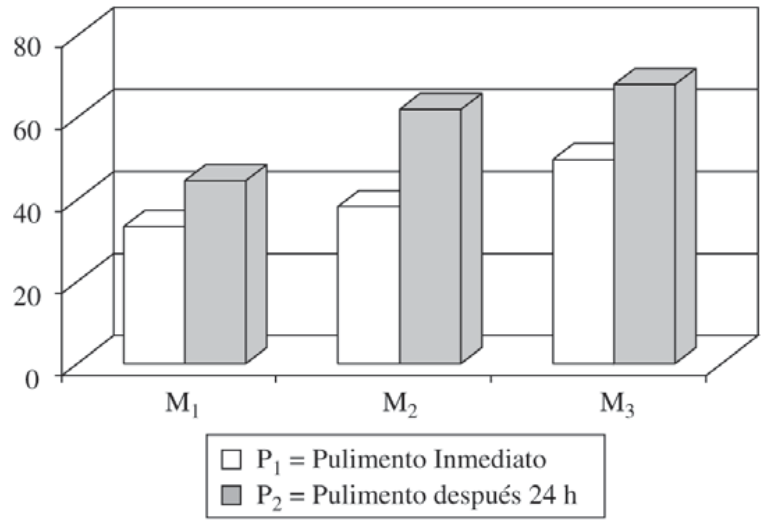

Figura 4. Interacción material $(\mathrm{M}) \times$ momento de pulimento $(\mathrm{P})$.

cial que la aleación Velvalloy (33,4 VHN) y ésta a su vez fue igual estadísticamente a la aleación Dispersalloy $(31,4 \mathrm{VHN})$. Ya para el pulimento realizado después de las $24 \mathrm{~h}\left(\mathrm{P}_{2}\right)$, las aleaciones Permite $(68,0 \mathrm{VHN})$ y Dispersalloy (61,9 VHN) presentaron medias de dureza superficial estadísticamente iguales entre sí y mayores que aquellas determinadas por la aleación Velvalloy $(44,6$ VHN). Fue observado también que cuando es realizado el pulimento inmediato de los especímenes confeccionados con la aleación Permite $(49,7$ VHN), la dureza superficial fue estadísticamente igual a la dureza correspondiente al pulimento realizado después de 24 h utilizándose la aleación convencional Velvalloy $\left(\mathrm{M}_{1}\right)$, lo que justifica esta interacción.

Analizándose las interacciones Material $(\mathrm{M}) \times$ Técnica de Pulimento $(\mathrm{T})$, y Momento de Pulimento $(\mathrm{P}) \times$ Técnica de Pulimento $(\mathrm{T})$, se verificó que no hubo efecto estadísticamente significativo de un factor sobre el otro. 


\section{Discusión}

El pulimento de la amalgama es un paso muy importante para que se obtenga el éxito clínico de una restauración, sea del punto de vista físico-mecánico, así como biológico. Este último está directamente relacionado a la liberación de mercurio. Varios factores influyen en el pulimento, tales como el tipo de aleación, momento y técnica de pulimento.

En el análisis del factor Material se observó que la aleación convencional (Velvalloy) determinó una menor dureza superficial que las aleaciones de alto contenido de cobre (Dispersalloy e Permite), resultado este concordante con trabajos realizados por Nakai et al. (1970), Prevedel \& Echazú (1986), Patsurakos \& Moberg (1988), Patsurakos \& Moberg (1990), Araújo et al. (1990), Ribeiro et al. (1991), Perdigoni et al., (1991). Eso, según estos autores se debe a la mayor cantidad de fase gama 2 presente en las amalgamas convencionales, que poseen una mayor tendencia a la corrosión y por lo tanto fragiliza la superfície de la amalgama, disminuyendo así la dureza superficial. La cantidad de mercúrio, según Perdigoni et al. (1991) también influye en la dureza, así las amalgamas que requieren menor cantidad de mercúrio para su trituración, el caso de la aleación Permite en este trabajo $(1: 0,97)$, determinó mayor dureza superficial cuando fue comparada con las de Dispersalloy y Velvalloy con proporción aleación/mercúrio de 1:1, además de eso, según Ilikli et al. (1999), la incorporación de indio (caso de la aleación Permite) aumenta la resistencia a la corrosión y por lo tanto también la dureza.

Con relación a la técnica de pulimento, se constató en este estudio que la técnica que utiliza instrumento cortante ( $\mathrm{T}_{1}=$ broca multilaminada, gomas abrasivas y piedra pomes) determinó menores medias de dureza que aquella que utiliza solamente instrumentos pulidores $\left(\mathrm{T}_{2}=\right.$ gomas abrasivas y piedra pomes), este resultado está de acuerdo con los trabajos de Araújo et al. (1990) y Ribeiro et al. (1991) siendo que éste último afirma que el uso de instrumentos rotatórios puede inducir a alteraciones mecánicas en la superfície de la restauración de amálgama.

Cuando se realizó el pulimento inmediato la dureza fue menor que cuando realizado después de las $24 \mathrm{~h}$, eso puede ser debido a la baja cristalización que las amalgamas presentan en el momento del pulimento inmediato, especialmente las amalgamas convencionales, las cuales determinaron menores niveles de dureza superficial que aquellas de alto contenido de cobre en las mismas condiciones.

En el análisis de la interacción Material $(\mathrm{M}) \times$ Momento de Pulimento (P), fue observado que las amalgamas confeccionadas con la aleación Velvalloy pulidos después de $24 \mathrm{~h}$ presentaron dureza estadísticamente igual a aquellas confeccionadas con la aleación Permite que recibieron pulimento inmediato. Demostrándose así, que es posible la realización del pulimento inmediato de las restauraciones de amalgama en una única sesión, desde que la aleación sea escogida de forma adecuada. Este resultado es importante, ya que en la literatura (Nitkin \& Goldberg, 1979; Yamashita et al., 1995; Pereira et al., 1998) se constata que este procedimiento es negligenciado por los odontólogos, que se justifican por la pérdida de tiempo que representa, pues necesitaría otra consulta para ejecutarla. La relevancia de este estudio está en mostrar la posibilidad de utilización segura del amalgama, sin comprometimiento de sus propiedades mecánicas, ni dejar de lado el aspecto biológico de su colocación en la cavidad bucal, que está directamente relacionado a la liberación de mercurio, siempre que se proceda a una selección adecuada del tipo de aleación y técnica de pulimento.

\section{Conclusiones}

De acuerdo con la metodología utilizada se concluye que:

- La aleación Permite determinó una mayor dureza superficial que la aleación convencional Velvalloy, permaneciendo en posición intermedia la aleación Dispersalloy;

- Amalgamas pulidas después de $24 \mathrm{~h}$ resulta en una mayor dureza superficial que aquellas pulidas inmediatamente;

- La no-utilización de instrumentos cortantes para el pulimento favorece una mayor dureza superficial.

- La aleación Permite pulida inmediatamente presentó dureza superficial igual que la aleación Velvalloy pulida después de $24 \mathrm{~h}$. Esto significa que la aleación Permite puede ser pulida inmediatamente sin comprometimiento de su dureza.

\section{Referencias}

1. Anusavice, K.J. Phillips: Materiais dentários. 10 ed. Guanabara Koogan, Rio de Janeiro, Brasil, 1998.

2. Araújo, M.A.J.; Fichman, D.M.; Muench, A.; Villela, L.C. Amálgama dental: Influência do polimento imediato na dureza superficial, rugosidade superficial e emissão de mercúrio residual - Estudo "in vitro". Rev Paul Odont, v. 12 , p. 46-59, 1990.

3. Canilo, M.H.; Centola, A.L.B.; Turbino, M.L. Análise rugosimétrica de restaurações com amálgama, Rev Facul Odont Lins, v. 9, n. 2, p. 6-11, 1996.

4. Drummond, J.L.; Jung H.; Savers, E.E.; Novickas, D.; Toepke, T.R.S. Surface roughness of polished amalgams, Oper Dent, v. 17, n. 4, p. 129-134, 1992.

5. Elizaur, A.B.C; Dinelli, W.; Gabrielli, F.; Mendes, A.J.D. Amálgama Dental. Estudo da formação da película de oxidação através do método de refletância aparente e análise microscópica, efeito do tipo de limalha, momento para execução do polimento e tempo, Odont Clín, v. 1, n. 4 , p. 7-14, 1987. 
6. Garone, N. Influência do alisamento na microestrutara superficial do amálgama dental, Rev Assoc Paul Cir Dent, v. 35, p. 427-438, 1981.

7. Ilikli, B.G.; Aydin, A.; Isimer, A.; Alpaslan, G. In vitro corrosion behaviour and microhardness of high-cooper amalgams with platinium and indium, J Oral Rehabil. v. 26, p. 170-176, 1999.

8. Nakai; H.; Ishizaki, N.; Nihei, I. The microestruture and hardness of dental amalgam, J Osaka D Univ, v. 4, n. 2, p. 131-149, oct, 1970.

9. Nitkin, D.A.; Goldbergh, A.J. Placing and polishing amalgam in one visit, Quint Int, v. 10, n. 6, p. 26-31, 1979.

10. Patsurakos, A.; Moberg, L.E. Corrosion behavior and microhardness of tree amalgams, Scand J Dent Res, v. 96, n. 4 , p. $376-383,1988$.

11. Patsurakos, A.; Moberg, L.E. Marginal microhardness of corroded amalgams: a comparative in vitro study, Scand J Dent Res, v. 98, n. 4, p. 326-335, aug, 1990.

12. Perdigoni, M.L.; Centola, A.L.B.; Froner, I.C.; Turbino, M.L.; Ribeiro, S.A. Effect of the polishing technique at low or high speed on the micro-hardness of dental amalgam, Braz Dent J, v. 2, n. 1, p. 51-57, 1991.

13. Pereira, M.A.; Centola, A.L.B.; Nascimento, T.N.; Turbino, M.L. Rugosimetric analysis of dental amalgam restorations polished using different techniques, $\mathrm{Braz}$ Dent J, v. 9, n. 2, p. 85-90, 1998.

14. Prevedel, V.; Echazú, A.M. Pulido y dureza superficial en amalgamas, Asoc Odontol Argent, v. 74, n. 1, p. 12-13, feb. 1986.

15. Ribeiro, S.A.; Nascimento, T.N.; Centola, A.L.B.; Teixeira, L.C.; Campos, S.M. Effect of polishing burs and stones on the micro-hardness of dental amalgam, Braz Dent J, v. 2, n. 2, p. 135-143, 1991.

16. Sundfeld, R.H.; Fontana, U.F.; Gabrielli, F.; Russo, M; Fontana, R.H.B.T.S.; Gomes, J.C.; Cabral, A.J. Avaliação clínica fotográfica da corrosão superficial, em restaurações de amálgama. Efeito de tipos de ligas, tratamentos superficiais, polimentos e tempos, Rev Odont UNESP, v. 18, p. 27-32, 1989.

17. Yamashita, C.; Capp, C.I.; Fichman, D.E.; Youssef, M.N. Estudo rugosimétrico da superfície de corpos-de-prova de amálgamas submetidos a diversas técnicas de polimento, Rev Pós Graduação, v. 2, n. 5, 1995. 